EPJ Web of Conferences 31, 00017 (2012)

DOI: $10.1051 /$ epjconf/20123100017

(C) Owned by the authors, published by EDP Sciences - SIF, 2012

\title{
Isospin effects in central heavy-ion collisions at Fermi energy
}

\author{
E. Galichet ${ }^{1,2}$, M.-F. Rivet ${ }^{1}$, F. Gagnon-Moisan ${ }^{1,3}{ }^{*}$, \\ B. Borderie ${ }^{1}$, M. COLONnA ${ }^{4}$ and R. RoY ${ }^{3}$ \\ (INDRA COLlaboration)
}

${ }^{1}$ Institut de Physique Nucléaire, CNRS/IN2P3, Université Paris-Sud 11, 91406 Orsay, France

2 Conservatoire National des Arts et Métiers, F75141 Paris

3 Département de Physique, Génie Physique et Optique, Université Laval, Québec, Canada

${ }^{4}$ LNS-INFN, I95123, Catania, Italy

\begin{abstract}
$\mathrm{N} / \mathrm{Z}$ effects were observed on variables measured in central Xe on Sn collisions around the Fermi energy. Comparisons were made with dynamical simulations of collisions in which the symmetry term of the EOS was varied. In this framework it appeared that the observed effects were due to the dynamics of the reaction and not to the deexcitation of the hot primary fragments.
\end{abstract}

The elaboration of the Equation of State (EOS) for nuclear matter requires more experimental constraints, particularly for the formulation of an adequate symmetry term whose density dependence at sub- and supranormal density is still debated. Isospin dependent observables measured in reactions induced by judiciously chosen projectile-target couples already provide some hints on the symmetry term; the derived form is however highly model-dependent. Thus cross-checks making use of a large body of variables and models are necessary to obtain reliable information.

\footnotetext{
${ }^{*}$ Current Address: Physikalisch-Technische Bundesanstalt, Bundesallee 100, D-38116 Braunschweig, Germany
}

This is an Open Access article distributed under the terms of the Creative Commons Attribution License 2.0, which permits unrestricted use, distribution, and reproduction in any medium, provided the original work is properly cited. 

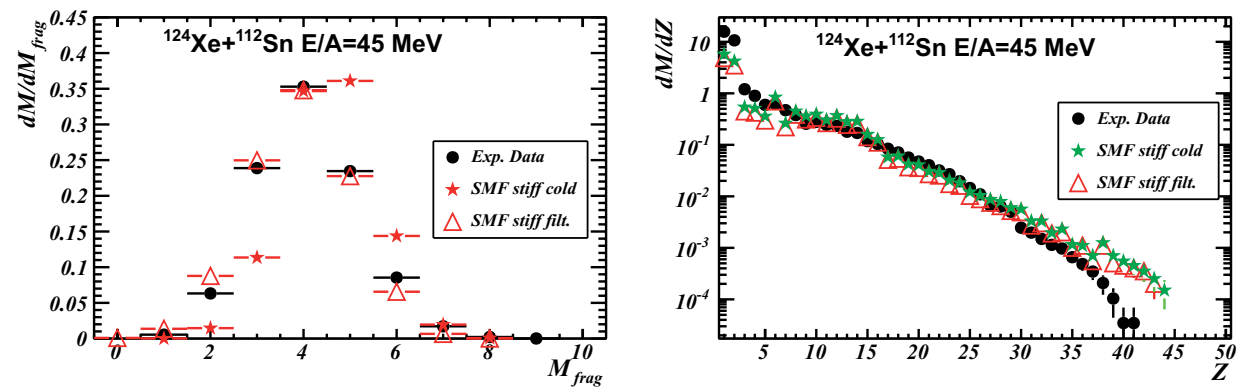

Figure 1: (Colour online) Comparison of experimental fragment multiplicity (left) and charge (right) distributions (circles) with calculated ones before (stars) and after (triangles) filtering.

Beams of 32 and $45 A \mathrm{MeV}^{124,136}$ Xe accelerated by the GANIL facility impinged on ${ }^{112,124} \mathrm{Sn}$ targets. Charged products were detected and identified with the $4 \pi$ INDRA array [1]. Compact shape events from central collisions were isolated by requiring that the sum of the charges of the detected products be at least equal to 80 and that the direction of the main axis of the kinetic energy flow-tensor with respect to the beam direction (in the reaction center of mass), $\theta_{\text {flow }}$, be greater than $60^{\circ}$. In the following, charged products with a charge $Z \geq 5$ are called fragments whereas those with $Z \leq 2$ are light charged particles $(l c p)$.

The evolution of various average multiplicities with the $\mathrm{N} / \mathrm{Z}$ of the total systems (projectile + target) has been shown in $[2,3]$. It was observed that the average charged product and $l c p$ multiplicities decrease when the system becomes more neutron-rich whereas the fragment multiplicity increases. For a given total system, the entrance channel mass asymmetry has no influence.

Head-on collisions $(b=0)$ were simulated with the stochastic mean field (SMF) calculation described in ref $[4,5]$; the same code was used in ref [6] to extract information on the symmetry energy from isospin diffusion. The isoscalar EOS is soft $\left(K_{\infty}=200 \mathrm{MeV}\right)$, and two parameterisations of the potential part of the symmetry energy are used, an asystiff one linearly increasing with density while the asysoft term ( $\left.\mathrm{SKM}^{*}\right)$ has a maximum at normal density. The free nucleon-nucleon cross-section, with its isospin, energy and angular dependence enters the collision term. With this version of the code the systems multifragment only at $\mathrm{E} / \mathrm{A}=45 \mathrm{MeV}$. Fragments are recognized through a density cut $\left(\rho>\rho_{0} / 5\right)$. The primary fragments present at 300 $\mathrm{fm} / c$, with their charges, masses, momenta, excitation energy and positions, were injected in the SIMON code [7] which performed the secondary decay 


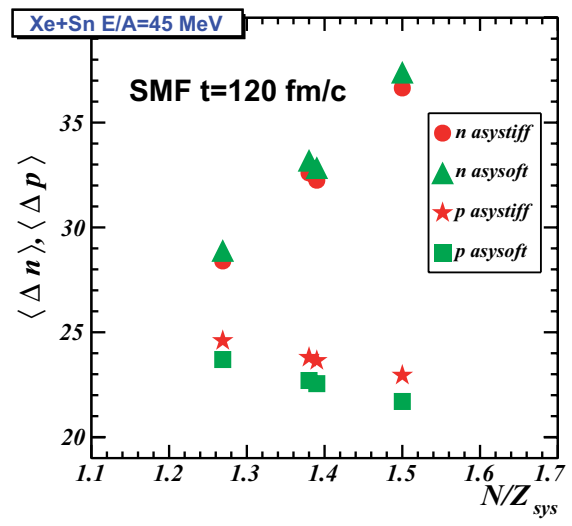

Figure 2: (Colour online) Number of preequilibrium neutrons (circles for asystiff EOS, triangles for asysoft) and protons (stars asystiff, squares asysoft) as a function of the system $\mathrm{N} / \mathrm{Z}$, at 45 $A \mathrm{MeV}$.

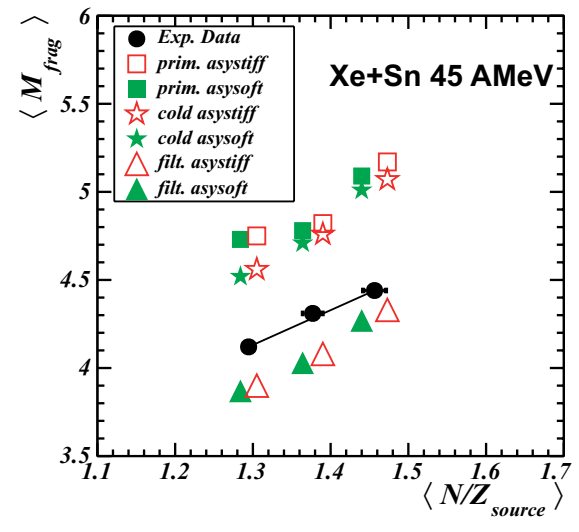

Figure 3: (Colour online) Average fragment multiplicities plotted versus the calculated $\mathrm{N} / \mathrm{Z}$ of the sources. Experimental data (circles), primary fragments (squares), cold fragments (stars) and filtered data (triangles); open symbols for asystiff EOS, filled ones for asysoft.

while preserving space-time correlations. Note that the fragment excitation energies at $300 \mathrm{fm} / c(\sim 3.3 \mathrm{AMeV})$ agree well with experimental determinations $[8,9]$. The cold products were finally filtered through a geometrical replica of the INDRA array described in the code PanForte [10]. Because particles in the gas phase are lost before the de-excitation step, only fragment related variables can be compared to the experimental values.

The simulation accounts reasonably well for the static variables (fragment multiplicity and charge distribution), as shown in fig. 1, but strongly underestimates the fragment kinetic energy, due to a late fragment formation and the lack of thermal fluctuations. Thus for the present time only variables related to multiplicities and charges will be used in model-data comparisons.

We define the multifragmenting source as the big fragment existing at $\mathrm{t}=120 \mathrm{fm} / c$. It is shown in [11] that the rate of nucleon emission in SMF is large up to that time and strongly decreases later on. The evolution of the number of preequilibrium nucleons (defined as the difference between the system and the source numbers of neutrons and protons) as a function of the $\mathrm{N} / \mathrm{Z}$ of the systems is shown in fig. 2. About 8-9 more neutrons are ejected by the neutron-richer system as compared to the neutron-poorer ( $1 / 3$ of the 24 extra-neutrons), whereas the latter system emits about two more protons. 

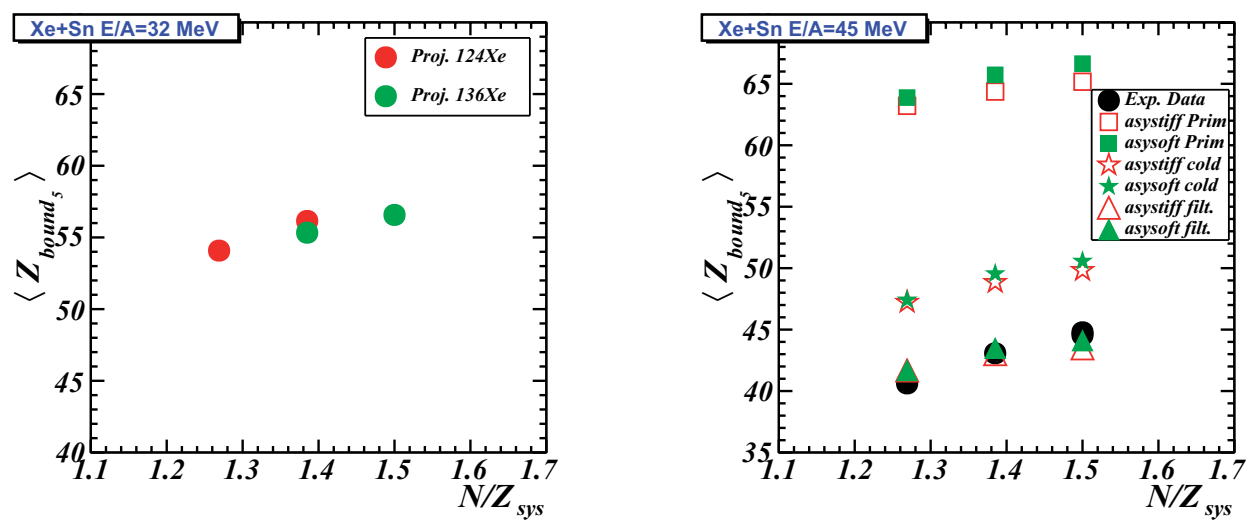

Figure 4: (Colour online) Average experimental $\mathrm{Z}_{\text {bound }}$ values versus the system $\mathrm{N} / \mathrm{Z}$ at $32 A \mathrm{MeV}$ (left). At $45 A \mathrm{MeV}$ (right) the experimental values (circles) are compared with the simulated ones for primary fragments (squares), cold fragments (stars) and filtered data (triangles). Open symbols asystiff, filled ones asysoft

With an asysoft EOS, there are more neutrons and less protons emitted than in the asystiff case. Indeed in the model most of that emission occurs during the expansion of the system, thus at subnormal density [11]; in that density region the symmetry potential is more repulsive for neutrons, and more attractive for protons in the asysoft case. For ${ }^{136} \mathrm{Xe}+{ }^{124} \mathrm{Sn}$ both the source and the primary fragments are, on average, more neutron-poor than the system. For ${ }^{124} \mathrm{Xe}+{ }^{112} \mathrm{Sn}$ the three $\mathrm{N} / \mathrm{Z}$ values remain closer. Thus globally, the explored $\mathrm{N} / \mathrm{Z}$ range becomes narrower after preequilibrium emission and even more after multifragmentation.

The average calculated fragment multiplicities measured at $45 \mathrm{AMeV}$ are plotted in fig. 3 as a function of the source $N / Z$, for the two EOS. We observe that the multiplicity of primary fragments increases for neutron-rich sources. This effect can thus be attributed to the dynamics of the multifragmentation process. The de-excitation stage barely modifies the average fragment multiplicities. The final (filtered) values agree within $5 \%$ with the experimental measurements (the experimental points are reported at the average $\mathrm{N} / \mathrm{Z}$ of sources obtained with the asystiff and asysoft EOS). Note that the dependence of $M_{\text {frag }}$ on the source $\mathrm{N} / \mathrm{Z}$ is linear, independently of the EOS, which indicates that $M_{\text {frag }}$ reflects the properties of the sources and not those of the initial systems.

Selecting $l c p$ or clusters which can be unambiguously qualified as preequilibrium particles is a real challenge. Moreover most of transport codes do not integrate coalescence for producing complex preequilibrium 
Table 1: Differences between the number of preequilibrium protons (at $\mathrm{t}=100 \mathrm{fm} / \mathrm{c}$ ) and the values of $Z_{\text {bound }_{5}}$ at different steps of the calculation, and measured (last column) for the systems ${ }^{136} \mathrm{Xe}+{ }^{124} \mathrm{Sn}$ and ${ }^{124} \mathrm{Xe}+{ }^{112} \mathrm{Sn}$.

\begin{tabular}{|l|c|c|c|c|c|}
\hline $\begin{array}{l}\text { EOS } \\
\text { asy }\end{array}$ & $\begin{array}{c}\text { SMF } \\
\text { Preeq. }\end{array}$ & $\begin{array}{c}\text { SMF } \\
\text { Prim. Frag. }\end{array}$ & $\begin{array}{c}+ \text { SIMON } \\
\text { Cold Frag. }\end{array}$ & $\begin{array}{c}+ \text { filter } \\
\text { Fin. Frag. }\end{array}$ & Exp \\
\hline & $\Delta \mathrm{p}$ & $\Delta Z_{\text {bound }_{5}}$ & $\Delta Z_{\text {bound }_{5}}$ & $\Delta Z_{\text {bound }}$ & $\Delta Z_{\text {bound }_{5}}$ \\
\hline stiff & -1.65 & 1.96 & 2.59 & 1.8 & $4.16 \pm 0.1 \pm 1$ \\
soft & -2.0 & 2.76 & 3.16 & 2.43 & $4.16 \pm 0.1 \pm 1$ \\
\hline
\end{tabular}

particles. An alternative could be to look at the complementary part of the proton preequilibrium emission, the "liquid phase"; this can be done in the present experiment because the selected events contain a large part of the total charge of the system. Therefore we have followed the total charge bound in fragments, $Z_{\text {bound }}=\sum_{1}^{M_{\text {frag }}} Z_{Z>5}$, as a function of the collision stage, and compared the final result of the calculations with the experimental data. The evolution of $\left\langle Z_{\text {bound }_{5}}\right\rangle$ with the system $\mathrm{N} / \mathrm{Z}$ is shown on fig. 4. At both incident energies $\left\langle Z_{\text {bound }_{5}}\right\rangle$ increases for neutron-rich system, reflecting the expected inverse trend with respect to the preequilibrium proton number shown in fig. 2. At $45 \mathrm{AMeV}$ the calculated values can also be reported. $\left\langle Z_{\text {bound }_{5}}\right\rangle$ is an increasing function of the $\mathrm{N} / \mathrm{Z}$ of the system for primary fragments, confirming that we are dealing with a dynamical effect. Table 1 shows that in addition the difference between the neutron-rich and the neutron-poor systems, $\Delta Z_{\text {bound }_{5}}$ is slightly larger after de-excitation whereas the filtering stage goes in the reverse direction. $\Delta Z_{\text {bound }_{5}}$ is larger with the asysoft EOS. The authors of ref [11] advise to be cautious about the calculated value of $Z_{\text {bound }_{5}}$, which depends on the fragment formation process (the spinodal decomposition in SMF strongly unfavours light fragments). Nevertheless the large measured value of $\Delta Z_{\text {bound }}$ makes it a promising variable for quantifying preequilibrium emission and giving new hints on the symmetry energy term of the EOS.

Odd-even effects in $\mathrm{Z}$ distributions were already observed in spallation [12], compound nucleus [13] reactions, but never in well-selected multifragmentation events. As visible in fig. 1, there is no obvious staggering in raw $\mathrm{Z}$ distributions.

By using the technique of ref [13], namely dividing the charge distributions by themselves after a five-points smoothing, the odd-even staggering becomes manifest. As in all other data, we observe a more pronounced staggering for the neutron-poor system. The staggering has been attributed 

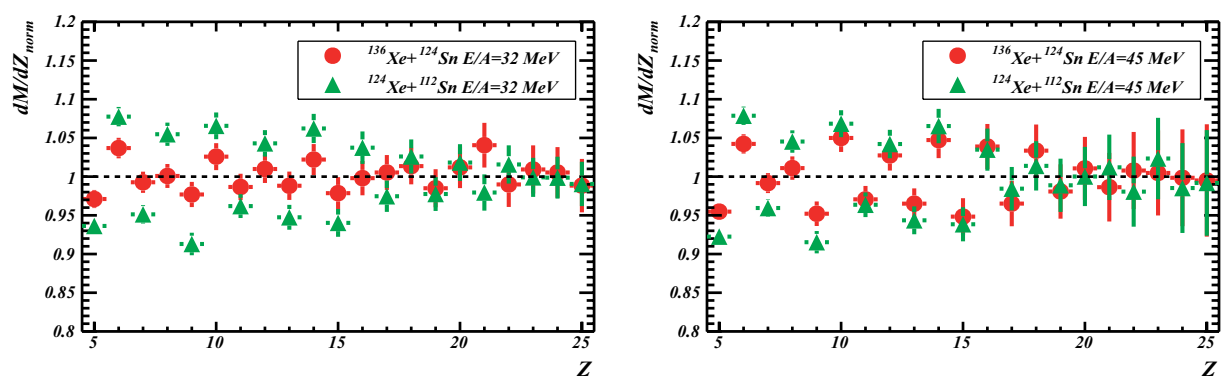

Figure 5: (Colour online) Normalized Z distributions for multifragmentation events (see text).

to the last steps of the de-excitation; its decrease for neutron-rich systems should be due to a reverse staggering for very neutron-rich fragments, whose yields are larger for neutron-rich systems. The difference between the neutron-poor and neutron-rich system is larger at $32 A \mathrm{MeV}$, which is difficult to understand as the fragment excitation energies are very similar at 32 and $45 \mathrm{AMeV}[8,9]$,

To summarize we observed N/Z effects in Xe+Sn central collisions which, according to the SMF simulations, are from dynamical origin :

i) Preequilibrium emission releases less protons for the neutron-rich system. We propose that the evolution of $Z_{\text {bound5 }}$ with system isospin would give an experimental hint on that emission and may give information on the symmetry term of the EOS.

ii) More fragments are produced in the multifragmentation of the neutronricher system. Entrance channel mass asymmetry does not influence the multiplicities.

iii) The $\mathrm{N} / \mathrm{Z}$ of the sources and primary fragments in the simulations strongly differ from those of the initial system for the neutron-rich system; for the neutron-poor system all N/Z values are closer. This observation has implications on the calorimetry calculations performed in experiments.

Finally we demonstrated that an odd-even staggering in the crosssections of elements produced in multifragmentation exists. As in all other observations the effect is less pronounced for neutron-rich systems. 


\section{References}

[1] J. Pouthas, B. Borderie et al., Nucl. Instr. and Meth. in Phys. Res. A 357 (1995) 418.

[2] F. Gagnon-Moisan, M. F. Rivet et al. (INDRA Collaboration), J. D. Frankland, A. Pagano et al. (eds.) Proc. Int. Workshop on Multifragmentation and related topics 2009, Catania, Italy, SIF Bologna, 2009, $165-170$.

[3] F. Gagnon-Moisan, thèse de doctorat, Université Paris XI Orsay et Université Laval Québec (2010), http://tel.archives-ouvertes.fr/tel00491182 .

[4] A. Guarnera, M. Colonna and P. Chomaz, Phys. Lett. B 373 (1996) 267.

[5] M. Colonna, M. Di Toro et al., Nucl. Phys. A 642 (1998) 449.

[6] E. Galichet, M. Colonna et al., Phys. Rev. C 79 (2009) 064615.

[7] D. Durand, Nucl. Phys. A 541 (1992) 266.

[8] S. Hudan, A. Chbihi et al. (INDRA Collaboration), Phys. Rev. C 67 (2003) 064613.

[9] S. Piantelli, B. Borderie et al. (INDRA Collaboration), Nucl. Phys. A 809 (2008) 111.

[10] P. Napolitani, M. Colonna et al., Phys. Rev. C 81 (2010) 044619.

[11] M. Colonna, A. Ono and J. Rizzo, Phys. Rev. C 82 (2010) 054613.

[12] M. V. Ricciardi, A. V. Ignatyuk et al., Nucl. Phys. A 733 (2004) 299.

[13] M. D’Agostino, M. Bruno et al., Nucl. Phys. A 861 (2011) 47. 